\title{
Characterization of XGM and XPM in a SOA-MZI using a Linear Frequency Resolved Gating Technique
}

T.T. Ng (1), A. Pérez (2), S. Sales (2), D.J. Richardson (1), P. Petropoulos (1)

1 : Optoelectronics Research Centre, B47, University of Southampton SO17 1BJ, ttn@orc.soton.ac.uk

2 : Optical and Quantum Communications Group, iTEAM Research Center, Universidad Politécnica de Valencia

\section{Introduction}

Semiconductor Optical Amplifiers (SOAs) are of considerable interest as nonlinear optical processing devices, since they can facilitate such operations as signal regeneration and wavelength conversion. All optical processing of signals in SOAs usually employs either cross-gain modulation (XGM) or cross-phase modulation (XPM). In the latter case, to convert phase changes to intensity variations, it is common to use integrated SOA Mach-Zehnder interferometers (SOA-MZIs). As signal repetition rates approach the operating bandwidth of the SOA devices, precise knowledge of their response in both intensity and phase becomes ever more crucial. The usual approach to the phase-sensitive characterisation of fast optical devices involves the use of interferometric set-ups [1], which can be quite involving and prone to environmental instabilities. In this paper, and in order to aid the optimisation of these versatile devices we present a study of SOA response behaviour using a linear frequency resolved gating technique (L-FROG). The L-FROG allows the phase and intensity of signals to be characterized using off-the-shelf all-fiberised equipment in a simple non-interferometric set-up. Using a SOA-MZI in a pump-probe configuration, we investigate both the XGM and XPM response to ps-long pump signals. Since intraband effects such as carrier heating and spectral hole burning, which facilitate high-speed switching, are dependent on the power of the incoming signals, we focus our studies on the variation of the SOA response to the input power. Through this study we hope to provide a better understanding of the nonlinear behaviour of SOA devices, and additionally prove the applicability of LFROG techniques for the complete characterisation of fast optical device response behaviour.

\section{Experimental Setup}

Our studies of SOA behaviour were performed on an integrated all-active, five-port SOA-MZI wavelength converter. This device has distinct $1 \mathrm{~mm}$ long SOAs placed in two different arms as shown at the top of Fig 1. Our XGM studies used only one of the SOAs in the SOA-MZI, whereas during our XPM studies we investigate the response of the device when both the SOAs were active.

The output was characterised using the L-FROG setup shown in Fig 1. A gain-switched DFB laser operating at $1550 \mathrm{~nm}$ (externally seeded by a cw source) was used to generate 7 ps pulses at a repetition rate of $10 \mathrm{GHz}$. The pulse train was then externally gated down to $5 \mathrm{GHz}$ to avoid patterning effects in the (dark) pulses to be characterized. The pulse train was split by a 50/50 splitter and detected by a fast $(32 \mathrm{GHz})$ optical detector to generate electrical gating pulses. These in turn were used to drive a $\mathrm{LiNbO}_{3}$ based Mach-Zehnder modulator (MZM) through which the SOA output signal was gated.

The spectrogram obtained by varying the optical delay in the gate arm of the L-FROG was then used in a blind deconvolution algorithm to retrieve the intensity and phase information of the SOA output signal [2]. A low-pass filter was applied to smooth out the gate function, thus improving the quality of the pulse retrieval. Note that since the L-FROG measurement uses the spectral information of the signal, a pump-rejecting optical filter is not required at the output of the SOA-MZI.

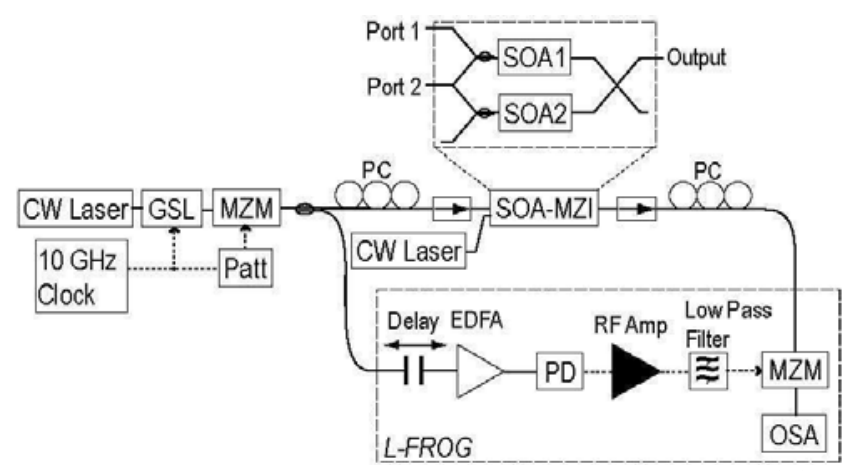

Fig. 1 - Experimental setup used to characterize the SOAMZI with the L-FROG technique

\section{Cross Gain Modulation}

XGM effects were investigated in SOA1 by illuminating it with the pulse train (pump) in Port 1 and a $1540.5 \mathrm{~nm} \mathrm{CW}$ probe (signal) in Port 2. A high current of $I_{1}=300 \mathrm{~mA}$ was used to induce a fast response time and large bandwidth in the SOA, and optimised to obtain an inverted output signal. We have measured the amplitude and phase of the signal at the output of the SOA-MZI. Fig. 2(a) shows the intensity and phase profile of the output signal for an average pump power of $-10 \mathrm{dBm}$. An inverted converted pulse, typical of the XGM process is observed. The phase profile shows that there is a large red shift (negative chirp) associated with the steep leading edge of the pulse and a smaller blue shift (positive chirp) associated with the shallow trailing edge of the pulse as a result of the slow response time. In Fig. 2(b) we see that both total and slow gain compression increase with increasing pump energy in agreement with theory [3]. The total gain compression increases with pump power largely 
because the plasma temperature in the active region is increased. At the same time the higher pump power increases stimulated emission, reducing carrier density and thus also increasing the slow gain compression [3].

The large difference between the total and the slow gain compression comes from the short pulse width. After the pump induced compression, the gain shows a fast recovery, resulting from the intraband effects. The slow interband recovery of the gain toward the unsaturated value then follows as a result of electrical pumping. Increasing the electrical bias current or the length of the SOA can shorten this recovery time [3]. For our $1 \mathrm{~mm}$ long SOA a period of 200 ps was sufficient to allow the gain to almost fully recover to its unsaturated value. Fig. 2(c) shows the trend in the dominating leading edge chirp with increasing average pump power. A variation of $80 \mathrm{GHz}$ is observed in the dominating negative chirp over a 7 $\mathrm{dB}$ variation in pump power. (a)

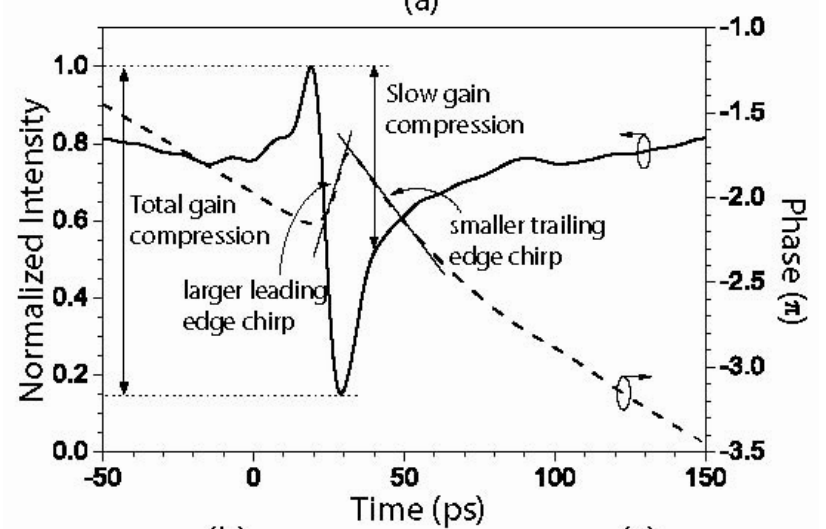

(b)
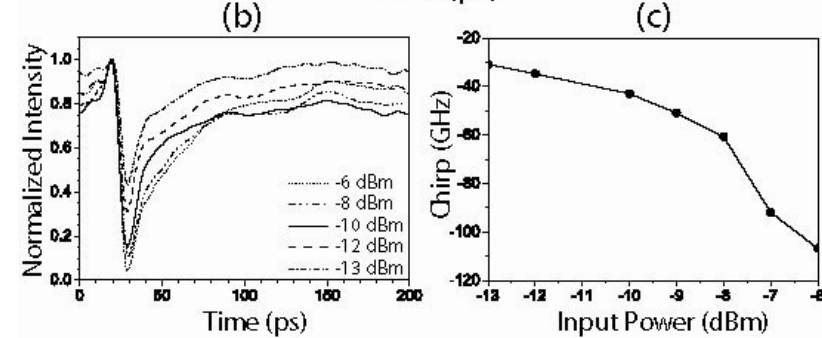

Fig. 2 - (a) Intensity and phase profile for average pump power of $-10 \mathrm{dBm}$ (b) Intensity profiles and (c) maximum chirp of the XGM pulses for different pump powers.

\section{Cross Phase Modulation}

We also measured XPM-based wavelength conversion in an interferometric set-up using both the SOAs. In this case average input powers between -6 and $-10 \mathrm{dBm}$ were used. The pump modulated the refractive index of SOA1, and hence the optical path length of the upper arm. This then caused destructive or constructive interference on the $\mathrm{CW}$ signal at the output. SOAs 1 and 2 were operated at current values of $\mathrm{I}_{1}=300 \mathrm{~mA}$ and $\mathrm{I}_{2}=160 \mathrm{~mA}$ respectively. These currents were selected for out of phase operation of the SOA-MZI to give inverted pulses.
Fig. 3(a) and (b) show the intensity profiles of the output signal with varying pump powers and the trend in their leading edge chirps. A continuous increase in the extinction ratio here suggests that a $\pi$ phase difference between the two arms was not yet reached. We expect that even higher pump powers would further increase the extinction ratio, though concerns of damaging the SOAs limited these measurements.

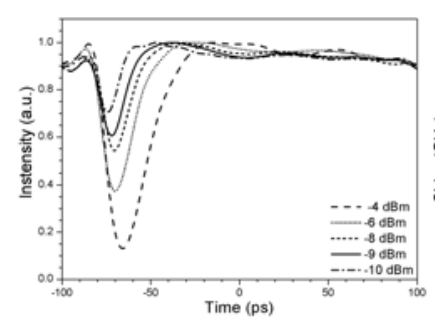

(a)

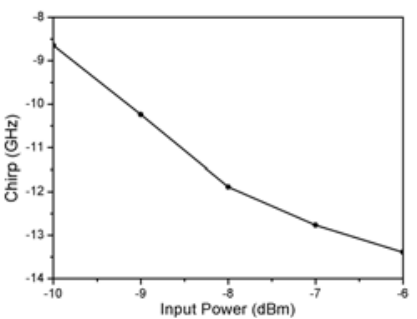

(b)
Fig. 3 - (a) Intensity profiles and (b) maximum chirp of the $X P M$ pulses for different pump powers.

We note that although trends between the XGM and XPM regimes are qualitatively similar, there is a significant difference between the chirp induced in the signal in these two regimes. In the XPM case, the maximum induced chirp is now much less than that observed in the XGM case. This occurs since the probe chirp is partially offset by its interference with a constant phase signal, and so is reduced by passing from SOA1 to the output of the MZI.

\section{Conclusion}

We have performed characterization measurements of $1 \mathrm{~mm}$ long SOAs operated in XGM and XPM configurations. We fully characterized the dynamic response of the SOA-MZI to 7 ps pulses at $5 \mathrm{GHz}$ using a L-FROG with a fast $\mathrm{LiNbO}_{3}$ Mach-Zehnder modulator as the sampling gate. Our measurements have provided experimental evidence of the dynamic nonlinear response of SOAs as predicted by theory [3] and allowed a direct comparison in the performance of the two nonlinear regimes. At the same time, our measurements demonstrate the applicability of the L-FROG technique for the characterization of the dynamic response of fast optical devices.

This work was supported by EU-NoE project ePhoton/One+.

\section{References}

[1] L.Schares et al, J. Quantum Electron., 39 (2003), p.1394 [2] B.E. Thomson et al, IEEE Photon. Technol. Lett., 17 (2005), p.1914

[3] L.Ochi et al, J. Quantum Electron., 38 (2002), p.54 